\title{
Tourisme et anthropologie : un tango de l'altérité
}

\section{Saskia Cousin and Thomas Apchain}

\section{OpenEdition}

\section{Journals}

Electronic version

URL: https://journals.openedition.org/tourisme/1320

DOI: 10.4000/tourisme.1320

ISSN: 2492-7503

\section{Publisher}

Association Mondes du tourisme

\section{Electronic reference}

Saskia Cousin and Thomas Apchain, "Tourisme et anthropologie : un tango de l'altérité", Mondes du Tourisme [Online], 12 | 2016, Online since 24 March 2017, connection on 21 September 2021. URL: http://journals.openedition.org/tourisme/1320 ; DOI: https://doi.org/10.4000/tourisme.1320

This text was automatically generated on 21 September 2021

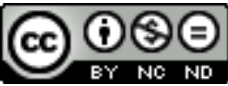

Mondes du tourisme est mis à disposition selon les termes de la licence Creative Commons Attribution - Pas d'Utilisation Commerciale - Pas de Modification 4.0 International. 


\title{
Tourisme et anthropologie : un tango de l'altérité
}

\author{
Saskia Cousin and Thomas Apchain
}

1 L'anthropologie du tourisme n'existe pas. Certes, il y a de nombreux travaux anthropologiques sur le tourisme, mais il n'y a, a priori, ni épistémologie ni corpus théorique communs : travailler sur les sociétés locales ou les touristes, sur les entreprises ou les politiques publiques, sur les réseaux virtuels ou les micro-interactions n'implique ni les mêmes méthodes, ni les mêmes théories. Toutefois, l'absence d'un cadre épistémologique stable pour l'analyse du tourisme en anthropologie ne tient pas uniquement à ces difficultés liées à la nature polymorphe de l'objet.

2 Ce sont d'autres facteurs que cet article entend pointer et analyser. Bien qu'il n'existe pas, pour les raisons évoquées, d'histoire théorique de l'anthropologie du tourisme, un simple retour historique sur l'évolution des relations entretenues entre la discipline et cet objet potentiel nous informe à la fois sur certains non-dits de l'anthropologie et sur les conditions qui pourraient permettre d'envisager une approche spécifiquement anthropologique du tourisme. Cette dernière se caractérise en effet par sa méthode ethnographique, c'est-à-dire par une immersion au sein de communautés relativement restreintes, soutenue par des observations menées sur un temps long. L'ethnologue s'intéresse aux pratiques et aux représentations des personnes avec qui sont menées les enquêtes. In fine, quelle que soit l'objet retenu (parenté, religion, pouvoir, jeux, etc.), l'enquête vise à décrire et analyser l'organisation des relations sociales propre à chaque collectif, et le sens qui lui est conféré.

3 Cet article propose donc de retracer l'histoire des recherches anthropologiques sur le tourisme, de présenter ses méthodes et ses questions, l'évolution de ses concepts et de ses objets, de cerner les relations complexes entre anthropologie et tourisme depuis l'origine jusqu'à ce que nous appellerons "l'ethnologisation» du tourisme. Nous tenterons d'identifier les problèmes scientifiques, académiques et épistémologiques propres à ces recherches. Par-delà la diversité des enquêtes et des approches, notre hypothèse est que la question de l'altérité, au cœur de la méthode anthropologique comme de la relation 
touristique, peut constituer le paradigme structurant d'une approche spécifiquement anthropologique du tourisme ${ }^{1}$.

\section{Comment les anthropologues se sont-ils (elles) saisi de la question du tourisme?}

Un retour historique sur les recherches anthropologiques sur le tourisme permet d'en situer l'origine: dans les années 1960, quelques études isolées, effectuées par des universitaires en majorité anglophones. Ces derniers ont peu à peu constitué une petite communauté de recherche, grâce notamment à la création de la revue Annals of Tourism Research et de commissions internationales. L'histoire de ces pionniers et de leurs recherches a été racontée par eux-mêmes (Nash, 2007). Ici, il s'agit plutôt de revenir sur les conditions particulières de l'émergence $d u$ tourisme comme objet pour l'anthropologie. L'état actuel de la recherche ne peut s'expliquer sans ce retour généalogique vers le moment où prennent racine des rapports particuliers entre l'anthropologie et le tourisme, et où se structurent, non sans problèmes, ses contours théoriques, méthodologiques, académiques et épistémologiques.

5 À l'instar, sans doute, de tout objet isolé ou mal aimé, le tourisme a été initialement étudié par des personnes qui se penchent sur ce thème en raison de leur histoire familiale ou de leur position sociale et académique. La première époque est constituée de travaux isolés, souvent redécouverts et considérés comme fondateurs a posteriori, comme La Classe de loisir de Thorstein Veblen (1899). Vient ensuite Theron Nunez, anthropologue à Berkeley qui publie dans Ethnology, en 1963, la première enquête ethnographique sur le tourisme, centrée sur les week-ends des riches Mexicains dans des villages traditionnels. À sa suite, plusieurs anthropologues se saisissent rapidement de cet objet: Nelson H. H. Graburn, Dennison Nash, Edward Bruner, Dean MacCannel, Jeremy Boissevain, Erik Cohen ou encore Malcom Crick et Jafar Jafari, fondateur de Annals of Tourism Research. Deux femmes: Valene Smith (1978), venue de la géographie, qui organise en 1974 la première session consacrée à l'anthropologie du tourisme à l'AAA (Leite et Graburn, 2010) ; Marie-Françoise Lanfant $(1972,1978)$, sociologue des loisirs, et unique française à explorer ce champ dès les années 1970.

6 Au cours de ces années 1970, la recherche anglophone s'est structurée à travers plusieurs revues, et en particulier Annals of Tourism Research, fondée en 1973 par l'anthropologue Jafar Jafari, avec le soutien de plusieurs anthropologues. Alors que la géographie domine les études françaises, l'anthropologie est donc l'une des disciplines fondatrices des recherches sur le tourisme dans le monde anglophone. Cette présence de l'anthropologie dès les origines permet de mieux comprendre l'évolution des recherches que Jafar Jafari résume dans un ouvrage collectif consacré aux fondements de l'anthropologie du tourisme : défense du tourisme comme moyen de développement dans les années 1960, puis, dès le début des années 1970, dénonciation des effets négatifs du tourisme dit « de masse» et valorisation des formes de tourisme jugées plus respectueuses des communautés hôtes (Nash, 2007). Enfin, dans les années 1990, une phase de production de connaissances sur un phénomène mondial, assortie de la recherche d'un cadre global d'analyse. Selon cet ouvrage, l'évolution de la recherche correspondrait aux différentes phases du rapport qu'entretiennent les institutions internationales avec le tourisme. Ainsi, l'évolution des recherches anthropologiques sur le tourisme parait-elle davantage 
structurée, en tout cas jusque dans les années 1990, au travers d'une posture idéologique, voire militante, plutôt qu'autour de la construction d'un cadre épistémologique indépendant.

7 Pour résumer, on peut constater que ce champ a été fondé, comme les autres, essentiellement par de jeunes hommes occidentaux anglophones, mais dont la particularité commune - que l'on retrouve chez leurs successeurs - est de n'être pas issus d'un milieu universitaire. En position de relative marginalité vis-à-vis du monde académique, ils ont sans doute trouvé dans le tourisme, objet marginal et non théorisé, un sujet de recherche adéquat. Les objets, les lieux d'enquêtes, les méthodes et les concepts qu'ils ont initiés restent encore très largement au cœur des recherches ethnographiques (Graburn, 2001). La traduction dans le premier numéro de Mondes du tourisme (Leite et Graburn, 2010) d'un extrait du grand article «Anthropological interventions in tourism studies» de Nelson H. H. Graburn et Naomie Leite (Leite et Graburn, 2009) donne un aperçu de la diversité des questions et des objets traités dès l'origine par les chercheurs anglophones: la question de l'impact du tourisme sur les sociétés traditionnelles, le corps et le genre des touristes, les arts (d'aéroport), l'authenticité, l'ethnicité, le patrimoine... Nous renvoyons à ce tour d'horizon précis et passionnant des recherches anglo-américaines pour nous focaliser ici sur les travaux plus récents, ou non évoqués parce que francophones. Nous évoquerons notamment le cas français et ses difficultés à consolider un cadre académique pour l'étude anthropologique du tourisme.

\section{Prise en compte tardive, dénonciation et mépris}

8 Jusqu'à la fin des années 1980, alors que bien des touristes sont déjà présents sur le terrain des anthropologues (en France comme ailleurs), la quasi-totalité des travaux des ethnologues ignore le phénomène. Les touristes et l'industrie du voyage sont «hors champ ", au sens photographique comme au sens sociologique du terme. Quelques très rares ouvrages se penchent toutefois sur la question, toujours dans le but de dénoncer les effets du tourisme sur les sociétés réceptrices. C'est le cas par exemple de l'ouvrage Le Tourisme en Afrique de l'Ouest: panacée ou nouvelle traite? paru en 1978 (Boutillier, Copans, Fieloux et alii). Cette vision manichéenne est omniprésente dans les articles et prises de position des intellectuels français de l'époque - «le tourisme, c'est mal!» - et dans les orientations d'institutions internationales comme le Conseil de l'Europe ou l'Unesco. Ces positions ont plusieurs explications. La première tient au constat de la déstabilisation de certaines sociétés par l'arrivée des touristes, jugement que nous viendrons à nuancer dans la mesure où l'on peut à la fois mettre en doute son universalité et en critiquer la vision essentialiste sous-jacente. La deuxième explication est liée au contexte international de l'époque. Les ethnologues s'opposent aux doctrines de la Banque mondiale édictées dans les années 1960, qui postulaient des effets automatiques de redistribution des richesses par le tourisme, dans le cadre des théories économiques du développement. Qu'ils suivent ou initient le rejet de ces doctrines, ils s'inscrivent dans le contexte de la lutte contre les impérialismes, de la conférence des non-alignés, de l'accès aux indépendances et du rôle joué par Moktar Mbow à la tête de l'Unesco (Cousin, 2008). Quelle que soit la légitimité de ce refus de l'imposition des doctrines et des flux, il reste que, contrairement par exemple à la revue Annals of Tourism Research qui adopte un point de vue critique mais informé, la plupart des études françaises ne dépassent pas le stade de 
la pétition de principe et ne cherchent pas à étudier concrètement les interactions entre les touristes et leurs hôtes. Malgré quelques études sur les loisirs et l'émergence de la sociologie de la culture, les années 1970 sont structurées par des écoles qui n'encouragent pas l'étude du temps libre et de la culture de masse. Si les chercheurs américains se confrontent tôt à la question, on est, du coté français et à l'exception des chercheurs de l'Uresti, passé d'une dénonciation politique à un mépris du phénomène. On peut supposer que ceci est également lié à la posture de rejet de la figure du touriste qu'analysera JeanDidier Urbain dans L'Idiot du voyage (1991). À l'évidence, le tourisme ne semblait pas faire partie des objets légitimes des sciences sociales. Par exemple, pour ce qui concerne l'histoire, Hasso Spode (2010) a bien montré que l'histoire du tourisme avait également été le fait de chercheurs isolés, à l'écart des sujets nobles et dominants. Plusieurs explications peuvent être avancées: sous-estimation de l'importance historique, économique et symbolique du tourisme, difficultés à appréhender un sujet mouvant, complexe et polymorphe, mépris pour un objet jugé trivial, voire vulgaire : loin de l'État et des grandes affaires culturelles, politiques ou religieuses, le tourisme serait une affaire de petits bourgeois, de petits commerçants, de petits territoires, et, donc, de petits chercheurs. Comment expliquer cela? Pourquoi les ethnologues français se sont-ils longtemps contentés d'un discours surplombant, dénonçant les effets pervers du tourisme sans en étudier localement les pratiques et les mécanismes ? L'existence d'effets pervers du tourisme est indéniable, mais il y a des effets de domination dans tous les éléments de la vie sociale (la religion, par exemple) sans que ceux-ci se trouvent ignorés par les anthropologues. Plus encore, il semble important de se saisir de tous les objets d'étude, surtout lorsqu'ils mettent en jeu des rapports de pouvoir et de domination, mais aussi des résistances et des appropriations.

\section{Le tourisme, part maudite de l'ethnologie?}

9 À ces hypothèses, communes à l'ensemble des sciences sociales, s'ajoute un questionnement propre à l'enquête ethnographique. Sur leurs terrains français, procédant a priori par induction, c'est-à-dire à partir des observations réalisées in situ, les ethnographes auraient dû voir les touristes et se saisir de cet objet avec la suspension du jugement nécessaire à toute enquête. Pourtant, ils n'ont pas voulu les étudier et ont par ailleurs systématiquement considéré que les sociétés qu'ils étudiaient n'étaient pas capables de résister au tourisme ou d'en faire quelque chose. On peut supposer qu'il y a là un zeste de paternalisme post-colonial et, surtout, un sentiment de dépossession et de détérioration de l'image de soi - en quelque sorte, les touristes sont venus polluer leurs (beaux) terrains et l'ethnologue se voit " poursuivi » par les touristes, qui lui présentent un miroir déformé de lui-même. Cette hypothèse semble validée par l'observation de la constitution de certains « hauts lieux du tourisme culturel » comme Bali (Picard, 1992) ou le pays dogon (Doquet, 2010). À propos de ce dernier, Marcel Griaule remarque en 1931 que les touristes suivent les ethnologues et détruisent le « fait ethnographique » (Griaule, 1931). Mais, s'ils les suivent, c'est que, comme le note Anne-Marie Thiesse (1991), « ce que le tourisme donne à voir [...] est bien souvent l'accommodement plaisant, sensuel et spectaculaire de savoirs produits dans une perspective érudite ou scientifique ». Comme le montre Anne Doquet (2016), les écrits de l'ethnologue alimentent le désir de connaître ces communautés culturelles : il devient, à son corps défendant, l'informateur du touriste cultivé. Cela semble insupportable à certains, car l'anthropologie française s'est en partie 
légitimée par une distanciation vis-à-vis du voyage et des voyageurs. C'est bien le sens de la fameuse phrase de Claude Lévi-Strauss qui introduit l'ouvrage Tristes Tropiques (1955): «Je hais les voyages et les explorateurs. " Ce que veut établir Claude Lévi-Strauss dès la première ligne d'un ouvrage dont il sait très bien que le lectorat sera plus éclectique que pour n'importe quel autre de ses écrits, c'est que l'anthropologie n'entretient avec l'acte de voyager qu'une relation de stricte nécessité; le voyage est un moyen pour la connaissance, et maudits soient ceux pour qui il est une fin en soi.

Lorsque le public cultivé s'est mis en quête des lieux et des communautés racontées par l'ethnographe, les ethnologues se sont donc mis à détester leurs lecteurs voyageurs, ces touristes venus désenchanter leur bel ouvrage. Impossible dans ce contexte d'en étudier les manières de faire, comme si la distanciation méthodologique était devenue impossible. Comme si le touriste était la part maudite de l'ethnologue, son double destructeur, et qu'approcher le tourisme comme objet, c'était risquer de devenir touriste. Or, il nous semble que c'est cette proximité, et non la distance, qui justement pose problème à l'ethnographe et l'empêche de se saisir de cet objet. Comme le touriste, l'ethnologue définit ce qu'il est dans l'ailleurs qu'il postule, par la comparaison permanente qu'il opère avec sa propre identité. Comme le touriste, l'ethnologue importe ses schémas d'analyse : c'est avec ses savoirs, sa culture, ses catégories qu'il regarde et désigne une altérité. D'ailleurs, pour de nombreuses populations, les ethnologues sont simplement une sorte de touristes (trop) curieux (Crick, 1989; Doquet, 2009), comme ils étaient, quelques décennies auparavant, des colons un peu spéciaux. Et force est de constater que les ethnologues classiques n'ont pas poussé très loin l'analyse des relations entre les colons et les communautés qu'ils étudiaient.

On peut bien sûr également mettre en avant des différences fondamentales - l'ethnologue reste, il revient, il écrit, etc. Toutefois, la clef de la compréhension du déni ou du rejet du tourisme se trouve bien dans la proximité plus que dans les différences. Il aurait pu être intéressant d'éclairer un peu les non-dits de l'anthropologie (Caratini, 2012), ses parts d'ombre. Non pas in fine dans l'objectif de mener une espèce d'auto-analyse anthropologique du rejet du tourisme par les anthropologues, mais afin de permettre de plus sereines mesures de ce que les sociétés et les pouvoirs font du tourisme et de ses images. Les relations entre voyage et ethnographie, et la filiation entre explorateurs, missionnaires, voyageurs, ethnologues et touristes, longtemps indicibles en France, ont été ailleurs très étudiées, notamment par James Clifford (1988), Malcom Crick, Nelson H. H. Grabrun et d'autres. Pour ce qui concerne les recherches francophones, Jean-Didier Urbain (1998) a ouvert la voie, avant d'être récemment rejoint par de nombreuses analyses réflexives (Winkin, 1996 ; Cousin, 2010 ; Doquet, 2016), notamment élaborées au cours du séminaire de recherche "Tourisme: recherches, institutions, pratiques", à vocation pluridisciplinaire, mais qui rassemble depuis 2004 la plupart des anthropologues qui travaillent sur ce sujet. Depuis dix ans, de nouvelles et nombreuses recherches sont produites par une nouvelle génération d'ethnologues moins travaillés par la défense de la grandeur de la discipline et la nostalgie de communautés et de terrains circonscrits. Alors que les recherches anglophones sont centrées sur la question des expériences et des imaginaires des touristes et de leurs destinations (Picard, 2011 ; Salazar, 2010 ; Salazar et Graburn, 2014), les anthropologues francophones s'inscrivent actuellement plutôt dans le champ de l'anthropologie politique, de l'étude des dynamiques et des enjeux de pouvoir. Pourtant, contrairement à leurs collègues anglophones ou hispanophones, la question postcoloniale, bien que sous-jacente, est rarement étudiée frontalement. On peut 
considérer qu'il s'agit de se démarquer des prédécesseurs français pour qui le tourisme devait être dénoncé sans nuance. Mais l'explication réside également dans la difficulté des sciences sociales françaises à appréhender la question (post)coloniale. Par ailleurs, la nouvelle génération française est essentiellement composée de chercheuses, ce qui reste un marqueur de la marginalité de la thématique, et l'accès à l'emploi est une exception: le chercheur ou la chercheuse française devait (doit?) changer de sujet ou de discipline pour intégrer une institution de recherche, à de rares exceptions près ${ }^{2}$. Il nous semblait important de rappeler cette histoire dans le contexte actuel où l'on peut observer un phénomène d'anthropologisation du tourisme, et parfois aussi de touristification de l'anthropologie.

\section{Problèmes épistémologiques}

12 Pour comprendre l'absence, puis l'émergence et l'évolution des études ethnographiques sur le tourisme, il ne suffit pas de donner une explication académique ou sociologique. Notre hypothèse est qu'il faut également prendre en compte des considérations théoriques. En effet, après une phase évolutionniste puis diffusionniste, les travaux se sont organisés, jusqu'aux années 1970 environ, autour d'un paradigme fonctionnaliste, puis structuraliste pour la France avec Claude Lévi-Strauss, et structuro-fonctionnaliste pour le monde anglophone, et en particulier britannique, avec Radcliff-Brown. Pour ce qui concerne l'ethnologie du proche, elle s'inscrit, pour la même période, dans la lignée des études de folklore qui se sont développées partout en Europe à partir du début du XIX siècle (Thiesse, 1999). Malgré leurs différences, ces multiples enquêtes, approches et théories comportent un point commun essentiel pour comprendre leur incapacité à étudier le tourisme: elles s'intéressent aux invariants, aux structures, aux solidarités collectives, bref à tout ce qui permet à une société de ne pas bouger, qu'il s'agisse d'un village bourguignon, basque, dogon ou guarani. Il y a ici une véritable différence en termes d'approches, de théories et d'objets entre cette ethnologie et la sociologie de la même époque, alors centrée sur les enquêtes sur le monde ouvrier et urbain depuis Le Play et Durkheim pour la France, et, bien sûr, l'école de Chicago pour les États-Unis.

Ceci explique l'absence de prise en considération du tourisme, et plus généralement des facteurs de modernité ou d'étrangeté par rapport à ce que les ethnologues considéraient comme les éléments consubstantiels à la communauté qu'ils étudiaient : pour la plupart, ils ont également ignoré la présence de l'islam et des Peuls en pays dogon, l'importance du Crédit agricole et des foires aux vins en Bourgogne, ou celle des remontées mécaniques dans les montagnes suisses. Sans parler des missionnaires, des multinationales et des ethnologues chez les Guaranis. On pense également à l'histoire de la célèbre photographie de Claude Lévi-Strauss qui, cadrée autrement par son collègue brésilien, montre l'arrière-plan devant lequel sont photographiés les Indiens: une mission équipée entre autres de lignes télégraphiques. Les "contacts zones" posent problème à l'anthropologie classique.

14 Pour travailler, ou simplement voir le tourisme, il faut accepter que les sociétés que l'on étudie interfèrent avec le reste du monde, changent, se transforment. Il faut accepter l'idée que les Jivaros rêvent peut-être aussi de touristes, et s'intéresser à ce qu'ils font de ces rêves, d'un point de vue pratique et politique. Finalement, seule l'école de Manchester ou le courant d'anthropologie dynamique initié par Georges Balandier pouvaient auraient pu - voir et étudier le tourisme, mais les touristes étaient alors peu visibles en 
Afrique, car la plupart d'entre eux étaient d'abord des cadres coloniaux. Il faut également sortir du cadre national en appréhendant la dimension internationale des pratiques, tout en suspendant un jugement de valeur sur la légitimité des pratiques touristiques. En France, ce point de vue est plutôt venu, au départ, d'une synthèse, portée par MarieFrançoise Lanfant et les chercheurs de l'Uresti, entre une critique de la sociologie des loisirs issue du marxisme et le postulat du loisir comme fait social total.

Le point commun entre les pionniers anglophones et leurs héritiers francophones réside toutefois dans l'attention au changement social, en même temps qu'il explique, en partie du moins, le décalage temporel de plusieurs décennies. En effet, quelle que soit la diversité de leur entrée dans l'étude du phénomène touristique, tous partagent un certain affranchissement vis-à-vis des théories et des écrits qui figent les populations non occidentales dans un rapport à la tradition et en gomment la contemporanéité. Mais il aura fallu trois décennies pour que certains anthropologues français parviennent à s'extraire à la fois de la chape structuraliste des études non occidentales et de l'héritage folklorisant de l'ethnologie de la France. Si l'on se penche sur l'itinéraire des anthropologues français qui ont initié les enquêtes ethnographiques sur le tourisme, ils ou elles viennent de la sociologie des loisirs (Marie-Françoise Lanfant) et de l'imaginaire (Jean-Didier Urbain, Rachid Amirou) pour les pionniers, de l'anthropologie politique et des études africanistes pour leurs successeurs, et n'avaient tout simplement pas, au départ du moins, conscience de la force du modèle structuraliste et des embûches professionnelles qui les attendaient, alors même que leurs collègues anglophones avaient vécu les mêmes déconvenues académiques quelques décennies auparavant. De la part des anthropologues, l'invisibilité du tourisme est donc liée à des questions épistémologiques, mais aussi idéologiques, car le point commun entre tous est également la violence du rejet dont ils ont été l'objet. Au cours des années 2000, les thèses sur le tourisme se sont toutefois multipliées en France, faisant émerger une génération de chercheurs, ou plus souvent de chercheuses, qui travaillent ensemble et sont au fait des publications internationales. Les numéros spéciaux dans des revues généralistes ont accompagné ou suivi (Roux, 2009) ce qui a fini par constituer un programme de recherche: Autrepart, Cahiers d'études africaines, Civilisations, Ethnologie française, Ethnologies, Cahiers des Amériques latines ${ }^{3} .$. Or, dans ces numéros, il faut bien admettre que nous réinventons les tâtonnements, les sujets, les objets et les préoccupations de nos aînés anglophones. Si on peut le regretter, cela confirme également l'idée que certains objets touristiques sont ethnographiquement bons à penser.

Pour résumer, le structuro-fonctionnalisme empêche de voir les touristes, tandis que la question de l'arrivée des touristes devient centrale lorsque l'on s'intéresse au changement social, notamment dans les sociétés dites traditionnelles, et en particulier les petites communautés rurales, avec parfois, sans doute, une surestimation de l'effet de ce tourisme sur les sociétés concernées. Il est, d'ailleurs, intéressant de noter que la touristification des communautés considérées comme anhistoriques par le structuralisme semble plus importante qu'ailleurs. L'anthropologie centrée sur les invariants culturels se heurte à la réalité des transformations sociales, entre autres celles du tourisme dont elle dénonce les effets déstructurants. Le paradoxe est que cette anthropologie dénonciatrice plus que critique reste la plus présente et la plus mise en avant par les institutions internationales, les médias, les musées et... la communication touristique. En effet, la dénonciation du tourisme est ce qui se « vend » le mieux au public cultivé. 


\section{L'« ethnologisation » du tourisme}

17 Comme le note Anne Doquet (2016), «si on tentait de superposer deux cartes sur papier calque, l'une pointant les sociétés ici nommées "ethnologisées" et l'autre représentant les hauts lieux du tourisme culturel, il y aurait fort à parier qu'elles coïncideraient en de nombreux points ». L'étude du tourisme culturel, de ses lieux et de ses slogans fait découvrir quelque chose d'assez troublant: non seulement les ethnologues sont les producteurs de "destinations » cataloguées comme "exotiques", mais on assiste à la réappropriation profane, sociale et commerciale des concepts anthropologiques. Alors que les générations précédentes ont théorisé des notions pour en faire des concepts puis des paradigmes, nous étudions actuellement les effets de rétroaction de ces concepts sur les politiques et les pratiques touristiques. L'exemple séminal est le couple potlatch / doncontre-don théorisé par Mauss, peu à peu institué en paradigme central de la discipline, avant de devenir ces dernières années un argument de vente pour le tourisme culturel. Même observation pour les héros de la discipline, et en particulier Claude Lévi-Strauss, devenu un étendard du voyage cultivé et "responsable», cité dans tous les guides. Comment expliquer qu'un concept écartant les échanges économiques et un auteur inventeur de l'opposition entre société chaude et froide, explicitement opposé au voyage, aient pu devenir le cœur de la mise en mot et en marché du tourisme culturel cultivé ? Ce paradoxe n'est que de façade. La théorie structuralo-fonctionnaliste, centrée sur les traditions, la solidarité et la recherche de l'équilibre, se désire et se vend plus que le changement social, ses enjeux de pouvoir et ses désenchantements. Au sein même des travaux menés par des anthropologues sur le tourisme, ce sont les synthèses totalisantes qui s'exportent le mieux, notamment dans les disciplines proches de l'anthropologie.

Les termes des sociétés non occidentales, recueillis et diffusés par les anthropologues au $\mathrm{XIX}^{\mathrm{e}}$ et au début $\mathrm{du} \mathrm{Xx}^{\mathrm{e}}$ siècles sont désormais utilisés par les médias. Les notions de rite de passage, de caste, de rituel, de don et de contre-don, de potlatch, de kula, de mana, de fait social total... ont trouvé un nouveau souffle avec la question du tourisme, le plus souvent non plus comme catégorie analytique, mais comme objet, voire comme destination touristique. C'est toute la complexité de l'anthropologie contemporaine en général et de l'anthropologie du tourisme en particulier: elle analyse des concepts indigènes qui sont souvent une reconfiguration de ce que Lévi-Strauss nommait la pensée sauvage (1962) par les catégories ethnologiques, missionnaires ou coloniales.

Aussi, cette récupération profane des concepts de l'anthropologie pose certains problèmes théoriques et pratiques. Les catégories d'origine anthropologique que l'on retrouve dans les discours touristiques sont-elles des catégories étiques ou des catégories émiques? Cette question impose une gymnastique permanente tant certaines de ces catégories oscillent entre émique et étique plutôt qu'elles ne franchissent définitivement une barrière rigide. En revanche, certaines notions autrefois solidement ancrées dans le vocabulaire de l'anthropologue semblent avoir connu une « touristification » irréversible, se vidant ainsi de leur valeur heuristique. Toutefois, l'importance émique de ces notions les rend tout aussi centrales pour l'étude du phénomène touristique, qui doit alors accepter leur passage de concept analytique à catégorie indigène. C'est ce que nous allons voir à travers deux notions - authenticité et ethnie - qui, loin donc de disparaître du fait de ce changement de statut, ont marqué les enquêtes et l'approche anthropologiques du tourisme. 
20 La place de la notion d'authenticité en anthropologie du tourisme témoigne bien de l'évolution de la posture adoptée par la discipline face à - ou peut-être dans ce cas grâce à - sa réappropriation profane par les différents acteurs du tourisme. En effet, il a été montré ailleurs (Bruner, 1995; Urbain, 2003 ; Cravatte, 2009; Leite et Graburn, 2009 ; Cousin, 2011 ; Salazar, 2013) que les anthropologues, en particulier en France mais pas uniquement, ont d'abord adopté la position de juge vis-à-vis des pratiques, expériences, représentations et objets du tourisme. De manière générale, leurs discours consistent alors à distinguer le «faux » du « vrai ». Et si le discours porte souvent sur l'objet ou la performance reproduits, et donc falsifiés, pour le touriste, le jugement moral n'est jamais trop éloigné et les catégories du « vrai » et du «faux » cachent souvent celles du "bon » (le voyageur) et $\mathrm{du}$ " mauvais » (le touriste). Quand il se donne pour mission d'évaluer l'authenticité, l'anthropologue se pose donc, vis-à-vis du touriste, comme une autorité à la fois morale et scientifique. Il est possible, du moins en partie, que cette obsession anthropologique pour l'authentification, qui renvoie au modèle structuro-fonctionnaliste évoqué plus haut et dont on avait dit qu'il avait sur le public un fort pouvoir d'attraction, se soit diffusé au sein du phénomène touristique. Quoiqu'il en soit, l'authenticité est une notion que l'on retrouve à de nombreux niveaux dans les discours et les pratiques touristiques, si bien que dès 1976, Dean MacCannell a fait de l'idée que le tourisme se définirait en premier lieu comme une quête d'authenticité la thèse centrale de son ouvrage fondateur. Si l'anthropologie veut parvenir à échapper à cette relation d'inspiration-concurrence avec le tourisme et adopter une posture plus apte à la compréhension du phénomène, elle se doit donc de transformer l'authenticité de concept analytique à catégorie indigène. Ce faisant, la discipline ouvre un champ d'étude dans lequel l'authenticité n'est plus à déterminer a priori mais résulte d'un type particulier de rapports que le touriste entretient avec son expérience ou avec l'objet de sa pratique, et qu'il s'agit d'analyser. Adoptant cette posture, l'anthropologie a d'ailleurs montré que l'authenticité telle qu'elle avait été initialement instrumentalisée par certains chercheurs ne recouvrait qu'une partie infime de l'authenticité telle qu'elle fait sens pour les protagonistes du tourisme. Le sens de l'authenticité s'élargit par rapport à celui du concept analytique - dans lequel elle se limite à une nature froide (Cohen, 2012) et objective -, à une catégorie indigène coconstruite, dynamique et existentielle (Selwyn, 1996 ; Wang, 1999). Plusieurs articles de synthèse racontent précisément cette évolution (Olsen, 2002 ; Cravatte, 2009 ; Cohen, 1988). Plutôt que de travailler à séparer le bon de l'inauthentique, l'anthropologie travaille désormais sur la manière dont les acteurs construisent cette notion structurante, entre autres, d'un certain rapport à l'altérité.

21 Il en va de l'authenticité comme de la notion d'ethnie, qui elle aussi semble s'être définitivement installée dans le vocabulaire touristique. Même si elle travaille depuis longtemps à déconstruire cette notion (Amselle et M’Bokolo, 1985; Barth, 1969), l'anthropologie s'est construite autour de l'analyse de groupes localisés fréquemment désignés, en particulier sur le continent africain, par le terme " ethnie ». Si, à travers les relations entretenues dans le passé avec l'administration coloniale, l'anthropologie a pu être créatrice d'ethnie, c'est surtout en tant qu'intermédiaire entre ces groupes et des lecteurs en quête d'exotisme que la discipline a constitué son rapport avec l'extérieur de la sphère académique. En effet, il est indéniable que l'anthropologie a pu, dans ses (rares) moments de diffusion profane, avoir un impact direct sur le phénomène touristique pour deux raisons. D'abord l'anthropologie a, quoi qu'il puisse être dit sur la manière, concouru à élargir la connaissance des hommes qui peuplent la planète et a constitué, 
pour de nombreux lecteurs, une invitation au voyage (notamment à travers les publications à grand tirage parmi lesquelles, chez Plon, les ouvrages de la collection "Terre humaine » ont eu un succès conséquent auprès du "grand public »). Ensuite, l'anthropologie a contribué, tout au long de son histoire, à produire des ethnies, des identités collectives identifiables ou, en tout cas, à les faire connaître à l'échelle mondiale (Salazar, 2013). Elle a ainsi diffusé une division ethnique du monde sur laquelle allait se calquer une labellisation de la diversité culturelle (idée de diversité dont on pourrait également, soit dit en passant, attribuer la paternité à l'anthropologie). C'est ce qui apparaît de manière générale dans le passage du lexique de l'ethnie dans le langage commun: "tourisme ethnique", "bijoux ethniques", etc. Là encore, tout en se modifiant, les catégories étiques sont devenues émiques et correspondent à une spatialisation du monde qui, n'ayant pas incorporé les critiques faites par l'anthropologie contemporaine, tend vers un essentialisme certain. Au succès du modèle structurofonctionnaliste s'ajoute donc celui du modèle culturaliste. Là encore, comme face à l'authenticité, l'anthropologie ne peut que constater cette porosité entre catégorie analytique et catégorie indigène, et abandonner ses velléités d'autorité sur la catégorisation de l'autre.

La réappropriation du langage de l'anthropologie par les différents acteurs du tourisme a des conséquences diverses sur la production scientifique. Nous pourrions, d'un côté, nous féliciter de cette diffusion profane, mais celle-ci s'accompagne, nous l'avons vu, de problèmes épistémologiques en ce qu'elle implique une confusion émique/étique et, donc, une complexification de l'impératif de distanciation. Cependant ce phénomène a peut-être, d'un autre côté, un avantage conséquent : il rappelle aux anthropologues la nécessité d'une approche inductive et constructiviste de l'identité et des mécanismes d'appréhension de l'altérité.

\section{L'altérité, paradigme central de l'anthropologie et du tourisme}

Le projet de l'anthropologie sociale peut se caractériser comme la discipline qui, à partir d'observations directes, vise à comprendre la manière donc chaque société, chaque communauté, et les personnes qui la composent, pensent leur relation au même et à l'autre. Ceci implique de s'intéresser à « l'autre de l'autre » (Bazin, 1985 ; Augé, 1994) dans une double dimension comparative : au sein d'une même société et entre les sociétés. Or, qu'est-ce que le tourisme, sinon la production et la consommation de "biens d'altérité ", c'est-à-dire de biens symboliques dont la valeur tient au sentiment d'altérité qu'ils suscitent?

La rupture entre sociologie et anthropologie s'est autrefois organisée à partir de la scission entre les sociétés traditionnelles non occidentales, rurales, aux solidarités mécaniques simples et de petite taille pour les anthropologues, et les sociétés industrielles, complexes, administratives, urbaines et aux solidarités organiques pour les sociologues. En subsiste encore une sorte d'atavisme ethnographique, à moins qu'il ne s'agisse d'une fascination exotique, qui explique que la majorité des enquêtes ethnographiques sont menées dans des sociétés non occidentales, rurales et considérées comme traditionnelles. La proximité des motivations touristiques et ethnographiques a été plusieurs fois étudiée (Crick, 1989 ; Urbain, 2003). Sans doute est-ce également parce 
que les médias, les musées, les promoteurs du tourisme culturel et la société industrielle en général, attendent de l'ethnologue des récits exotiques propres à être exposés, à faire rêver ou à faire consommer. Toutefois, cette répartition des terrains n'est plus valable d'un point de vue scientifique, épistémologique ou méthodologique, et cela fait un demisiècle que les sociologues et les anthropologues revisitent leurs terrains réciproques. Ce n'est pas le degré de traditionalisme ou d'intégration dans la globalisation qui détermine le caractère anthropologique d'une enquête. C'est donc ailleurs que se trouve la spécificité de son projet scientifique. Si l'enquête ethnographique et le projet comparatif sont également au cœur de la sociologie, les questions que le chercheur se pose ne sont pas les mêmes. Par exemple, le sociologue sera plus enclin à s'intéresser aux fonctions sociales et économiques de l'activité touristique, à partir d'hypothèses portant sur le tourisme et la société globale, « testées » sur un cas particulier, tandis que l'ethnologue se penchera sur les dynamiques internes, les transformations et les échanges en cours dans la communauté constituée par sa relation à ce que ses membres considèrent eux-mêmes comme du tourisme ou des touristes. Ceci implique plusieurs choses, quels que soient l'objet, le sujet et le terrain considérés.

Tout d'abord, une posture méthodologique de type inductive : par exemple, ne pas définir a priori un touriste, ne pas supposer que la pratique touristique serait mue uniquement par un désir de découverte ou de reconnaissance sociale, ou que le désir d'accueillir des touristes relèverait simplement d'une rationalité économique. En général, tous ces a priori s'effondrent d'eux-mêmes lorsqu'on accepte de renoncer à ces prénotions et que l'on fait l'effort de mener une enquête ethnographique. Ceci n'est pas simple à mettre en œuvre puisque cela implique une présence physique longue ou répétée sur son "terrain», ce que l'on appelle observation participante, ou directe. Il s'agit alors par exemple d'apprendre la langue locale (une langue étrangère ou une manière particulière de dire l'être-ensemble), et d'obtenir la confiance de la communauté avec laquelle on travaille... qu'il s'agisse d'une famille mongole (Merli, 2009) ou kirghize (Pabion Mouriès, 2010) qui accueille des touristes, de guides en pays dogon (Doquet, 2009), en pays lobi au BurkinaFaso (Chabloz, 2007), ou encore en Tanzanie (Salazar, 2006), de paysans du monde rural français (Abram, 1997; Rogers, 2002 ; Cousin, 2011), de la transformation de Majorque (Waldren, 1996) ou d'un groupe de trekkeurs en route pour l'Himalaya (Boutroy, 2006). Maïté Boullosa (2010), Katia Boissevain (2010) et d'autres ont particulièrement travaillé sur la patrimonialisation et la mise en marché touristique des folklores, des fêtes traditionnelles, votives ou religieuses, des pèlerinages. On s'intéresse aux manières de faire et de dire l'étranger, de construire le même et l'autre, dans les échanges de toute nature. Que l'on travaille très loin de chez soi ou dans un quartier voisin, ce qui compte alors, c'est le rapport d'altérité que l'on parvient à établir et maintenir avec son lieu et son objet d'enquête. En effet, si l'anthropologie et l'ethnologie se sont historiquement constituées dans une relation objective d'altérité - sociale, culturelle, linguistique, religieuse, etc. -, cette distance est aujourd'hui une construction méthodologique et épistémologique. Il faut pouvoir s'étonner de tout pour le voir, le noter et, plus tard, l'analyser. Lorsque la manière de penser des personnes avec qui l'on travaille devient une évidence, il est temps de quitter son terrain. Cette méthode permet de porter un regard décalé sur le phénomène touristique en s'intéressant plus particulièrement à ses dimensions symboliques et au sens que les humains en société donnent à ce qu'ils font dans une situation qu'ils considèrent comme relevant du tourisme. 

priori de définition du tourisme, mais nous étudions la valeur et le sens que donnent les gens à ce terme (Urbain, 1991), le rôle que chaque société lui confère, et la manière dont, in fine, il constitue un indicateur des rapports à l'identité et à l'altérité de chacun et de tous. Dans cette perspective, les catégories produites par les institutions, les entreprises, les ONG ou les autres disciplines des sciences humaines et sociales ne nous intéressent qu'en tant que catégories situées, dont nous étudions la circulation et les effets. Pour le dire autrement, si l'on pose la question : «Qu'est-ce qu'un touriste? » ou « Qu'est-ce que le tourisme? ", on ne répond pas de la même manière si on est une hôtelière de la Costa Brava, un paysan dogon, une élue berrichonne ou une statisticienne de l'Insee. Or, c'est la différence entre ces réponses qui nous intéresse : la manière dont les acteurs construisent des catégories, en discours comme en pratique. Par exemple, les catégories habitants / touristes sont omniprésentes dans les discours mais ne sont pas pertinentes lorsqu'on s'intéresse à la réalité des pratiques et des représentations de ce qui fait identité ou altérité pour les uns et les autres. Or, de ce point de vue, toutes les réponses sont légitimes pour l'anthropologue: son travail consiste à prendre de la distance avec chacune des catégories présentées afin d'en comprendre le sens et le rôle pour chaque communauté. Ceci n'est pas propre à la question du tourisme mais à l'anthropologie en général. Par exemple, si on travaille sur la parenté, on ne donne pas une définition unique et préalable de la parenté, mais on étudie ce que veut dire être parent, la manière dont les humains ont construit différemment la notion de parenté et ce que cela implique en termes de relations sociales, de construction de la personne, etc. catégories indigènes (émiques) implique aussi d'en reconstruire le sens avec les questionnements qui sont propres à la discipline. C'est par exemple ce que fait David Picard dans son ouvrage Tourism, Magic and Modernity (2011), dédié à la production de l'imaginaire des îles et de l'identité créole en contexte touristique. Il " prend au sérieux » la notion de magie utilisée par les touristes pour relater leur expérience tropicale en l'interprétant comme une consommation moderne mais rituelle de "jardins humains " insulaires.

De manière plus générale, un ou une anthropologue observe des pratiques sociales, religieuses, économiques, se penche sur la culture matérielle, le rapport au corps, aux images et au monde extérieur, écoute des récits, des discours, des échanges formels et informels. Il ou elle en déduit des valeurs, des catégories, des représentations de soi, de l'autre, du monde. Il ou elle les théorise avec la boite à outils qui lui est propre. Bref, il ou elle se penche sur le sens que les gens - touristes ou hôtes - donnent à ce qu'ils font euxmêmes et à ce que font et disent celles et ceux qu'ils considèrent comme proches ou différents. Qu'il s'agisse de l'identité des individus ou de la définition du tourisme, de ce qui est « authentique » pour les uns, et artificiel pour les autres: si l'anthropologue a un seul postulat, c'est que l'un et l'autre n'existent pas de manière intrinsèque mais se construisent en relation, en différenciation (le tourisme peut se définir par de multiples oppositions, avec la villégiature, le travail, le quotidien ou avec le « voyage », la culture... ). Pour l'anthropologue, le tourisme n'est donc pas défini, ni a priori ni a posteriori, mais il est dessiné par les discours, les pratiques et les représentations des personnes qui en tracent les contours, c'est-à-dire aussi, les frontières. C'est la manière dont se construisent ces frontières qui nous intéresse (Barth, 1969). Ce qui est pertinent pour l'ethnologie est moins le tourisme que ce qu'il révèle des relations sociales, dans leurs 
permanences mais aussi et surtout dans leurs variations et leurs différences. De fait, l'ensemble des problèmes épistémologiques, scientifiques et académiques que rencontre l'anthropologie confrontée au phénomène touristique tient aussi, du moins en partie, de la difficulté à considérer le tourisme comme une activité sociale fondamentale ou, à la manière des chercheurs de l'Uresti, comme un «fait social total». Nous pensons que le tourisme est l'une des expressions contemporaines des pratiques, des productions et des représentations symboliques de l'altérité, lesquelles sont bien au fondement des activités humaines. Le jeu social qu'il implique mérite que les anthropologues s'y penchent.

\section{Conclusion}

Dans cet article, nous avons évoqué les obstacles intellectuels qui rendent complexe l'existence même d'une anthropologie du tourisme. La méthode ethnographique et l'approche anthropologique nous paraissent en revanche aussi sous-employées que possiblement fécondes pour étudier certaines dimensions de cet objet. Que reste-t-il donc à faire pour qu'un champ spécifique émerge de l'analyse anthropologique du tourisme et que, par conséquent, se définisse l'apport de la question pour la discipline? Sans dresser un agenda exhaustif, nous pouvons évoquer, pour conclure, quelques points généraux sur lesquels des enquêtes restent à mener pour que le tourisme devienne un objet bon à penser pour l'anthropologie.

L'ethnographie du tourisme et de ses pratiques quotidiennes semble lacunaire en plusieurs points. La question de la culture matérielle des touristes est ainsi presque totalement absente des études francophones, ce qui empêche l'étude de la production et de la mise en circulation d'objets qui passent d'un usage quotidien ou rituel à d'autres statuts : artisanat, œuvre, « objet de musée » ou art " pour touristes ", avec des effets de rétroaction, étudiés par quelques anthropologues américains (Graburn, 1976; Bunten, 2008). La question du rôle du tourisme dans la construction des identités personnelles, sociales ou collectives ne peut être restreinte aux études - par ailleurs passionnantes - du tourisme généalogique en Irlande (Legrand, 2006) ou du tourisme des racines en Afrique de l'Ouest : par exemple, les vacances sont des pratiques au cœur de la production de la famille occidentale tandis que le voyage sans parents est un marqueur fort du passage à l'âge adulte. L'ethnographie du genre en situation touristique reste à faire, alors que les études se focalisent sur les rapports sociaux de sexe et le tourisme sexuel (Roux, 2011; Simoni, 2008 ; Salomon, 2009). Initiées dans les années 1980, les enquêtes sur les touristes non occidentaux se focalisent sur l'Asie (Graburn, 1995 ; Nyiri, 2006). Les ethnologues ont du travail pour comprendre la manière dont chaque société appréhende et s'approprie, à travers ses pratiques et ses imaginaires, l'injonction mondiale à la mise en mobilité.

Toutefois, l'angle mort des enquêtes est ailleurs : centrées sur l'arrivée du tourisme sur les terrains traditionnels des ethnologues, les ethnographies portent sur le tourisme culturel, solidaire, humanitaire, alternatif. Ni l'industrie (hébergements, transports, etc.) ni les grands organismes internationaux (Organisation mondiale du tourisme, OMC, etc.) liés au tourisme n'ont fait l'objet d'enquêtes ethnographiques. Or, travailler sur les objetssouvenirs, sur une institution internationale, sur un grand groupe hôtelier et plus encore, peut-être, sur les plateformes dites collaboratives (Couchsurfing, AirBnb, etc.) peut permettre de revisiter les paradigmes classiques de l'anthropologie économique. Dans une perspective pluridisciplinaire, c'est ce qu'ont commencé à faire David Picard et Sonja Buchberger (2013) pour Couchsurfing, Gael Chareyron, Saskia Cousin, Jérôme Da-Rugna et 
Sébastien Jacquot pour TripAdvisor et AirBnb (2014). En effet, la séparation théorique entre, d'un côté, les échanges marchands, contractuels, et, de l'autre, ceux gouvernés par le don-contre-don, la confiance, le prestige, n'est plus efficiente pour penser la transformation des pratiques, des économies et des valeurs symboliques à l'œuvre dans le tourisme. Par exemple, avec les échanges d'appartement, il n'y a plus de solution de continuité entre les hospitalités marchandes et non marchandes, les espaces domestiques et commerciaux, les hôtes et les professionnels de l'accueil. Enfin, l'ethnographie des circulations touristiques, des réseaux, des communautés numériques est balbutiante. Qu'il s'agisse des migrations ou des mobilités touristiques, des hommes, des objets ou des savoirs, des imaginaires ou des stéréotypes, l'observation de ces circulations est un défi méthodologique, et le tourisme une entrée toute légitime. Car, in fine, qu'est ce que le tourisme du point de vue de l'anthropologie? On pourrait le définir comme une « économie de l'altérité ». En effet, la valeur de ce qui s'échange en contexte touristique tient à la relation, à la pratique, ou à l'imaginaire d'altérité suscité par ce contexte. En conclusion, le phénomène touristique dans sa diversité sera mieux étudié si les ethnologues s'y penchent sérieusement, avec le savoir particulier des enquêtes localisées et multisites. Inversement, l'anthropologie a tout à gagner à étudier, de manière réflexive, le tourisme, ses réseaux et ses échanges, ses pratiques et ses imaginaires de l'autre, bref, ce «bien d'altérité » de notre temps.

\section{BIBLIOGRAPHY}

Simone Abram, « Performing for tourists in rural France ", dans Simone Abram, Jacqueline Waldern et Donald V. L. Macleod (dir.), Tourists and Tourisms: Identifying with People and Places, Berg, 1997, p. 29-50.

Rachid Amirou, Imaginaire touristique et sociabilités du voyage, Presses universitaires de France, 1995.

Jean-Loup Amselle et Elikia M'Bokolo (dir.), Au cœur de l'ethnie : ethnie, tribalisme et État en Afrique, La Découverte, 1985.

Anthropologie et Sociétés, vol. 25, $\mathrm{n}^{\circ} 2$ : « Tourisme et sociétés locales en Asie orientale », 2001.

Marc Augé, Pour une anthropologie des mondes contemporains, « Champs ", Flammarion, 1994.

Autrepart, vol. 40, $\mathrm{n}^{\circ} 4:$ « Tourisme culturel, réseaux et recompositions sociales » (dir. Sara LeMenestrel et Anne Doquet), 2006.

Fredrik Barth, Ethnic Groups and Boundaries: the Social Organization of Culture Difference, Little Brown and Company, 1969.

Jean Bazin, «À chacun son Bambara », dans Jean-Loup Amselle et Elikia M’Bokolo (dir.), Au cœur de l'ethnie, tribalisme et Etat en Afrique, La Découverte, 1985, p. 87-125.

Jeremy Boissevain (dir.), Coping with Tourism: European Reactions to Mass tourism, Berghahn Book, 1996. 
Katia Boissevain (dir.), « Nouveaux usages touristiques de la culture religieuse au Maghreb. Approches anthropologiques », dans Katia Boissevain et Pierre-Noël Denieul (dir.), Socioanthropologie de l'image au Maghreb, « Maghreb et sciences sociales » (2009-2010), L'Harmattan, IRMC, 2010.

Maïté Boullosa-Joly, « Tourisme, patrimonialisation et politique. Un cas d'école : la "Fête nationale de la Pachamama" (Nord-Ouest argentin) », Cahiers des Amériques latines, n 65, p. 103-120, 2010.

Jean-Louis Boutillier, Jean Copans, Michel Fieloux et alii, Le Tourisme en Afrique de l'Ouest : panacée ou nouvelle traite?, « Dossiers africains », François Maspero, 1978.

Éric Boutroy, «Cultiver le danger dans l'alpinisme himalayen », Ethnologie française, vol. 36, $\mathrm{n}^{\circ} 4$, 2006, p. 591-601.

Edward M. Bruner, « The ethnographer/tourist in Indonesia », dans Marie-Françoise Lanfant, John B. Allcock et Edward M. Bruner, International Tourism. Identity and Change, Sage, 1995, p. 224-241.

Alexis Bunten, « Sharing culture or selling out? Developing the commodified Persona in the heritage industry », American Ethnologist, vol. 35 (3), 2008, p. 380-395.

Cahiers des Amériques latines, $\mathrm{n}^{\circ} 65$ : Maïté Boullosa, Magali Demanget et David Dumoulin Kervran (dir.), « Tourisme patrimonial et sociétés locales en Amérique latine », 2010, p. 19-122.

Cahiers d'études africaines, $\mathrm{n}^{\text {os }} 193-194$ : « Tourismes : La quête de soi par la pratique des autres » (dir. Nadège Chabloz et Julien Raout), 2009.

Sophie Caratini, Les Non-dits de l'anthropologie. Suivi de Dialogue avec Maurice Godelier, « Les nondits ", T. Marchaisse, 2012

Nadège Chabloz, « Le malentendu. Les rencontres paradoxales du "tourisme solidaire" ", Actes de la recherche en sciences sociales, vol. 170, 2007, p. 32-47.

Civilisations, vol. 57 (1-2) : « Tourisme, mobilités et altérités contemporaines » (dir. Anne Doquet et Olivier Evrard), 2008.

James Clifford, The Predicament of Culture: Twentieth-Century Ethnography, Literature and Art, Harvard University Press, 1988.

Erik Cohen, « Authenticity and commoditization in tourism », Annals of Tourism Research, vol. 15, n o 3, 1988, p. 371-386.

Erik Cohen, « Authentification : hot and cool », Annals of Tourism Research, vol. 39, n 3, 2012, p. $1295-1314$

Saskia Cousin, "L'Unesco et la doctrine du tourisme culturel. Généalogie d'un "bon tourisme" ", Civilisations, vol. 57, $\mathrm{n}^{\text {os }} 1-2,2008$, p. 41-56.

Saskia Cousin, «Authenticité et tourisme? ", Les Cahiers du Musée des confluences, vol. 8 : « L'authenticité », 2011, p. 59-66.

Saskia Cousin, Gaël Chareyron, Jérôme Da-Rugna et Sébastien Jacquot, « Étudier TripAdvisor. Ou comment Trip-patouiller avec les cartes de nos vacances », Espacestemps.net, « Dans l'air », 2014. En ligne [consulté le 28 février 2017] : http://www.espacestemps.net/articles/etudiertripadvisor/

Céline Cravatte, «L'anthropologie du tourisme et l'authenticité : catégorie analytique ou catégorie indigène ?», Cahiers d'études africaines, vol. 193-194, 2009, p. 603-619. En ligne [consulté le 28 février 2017] : https://etudesafricaines.revues.org/18852 
Malcolm Crick, « Representations of international tourism in the social sciences », Annual Review of Anthropology, vol. 18, 1989, p. 307-344.

Anne Doquet, « “Guides, guidons et guitares”. Authenticité et guides touristiques au Mali », Cahiers d'études africaines, vol. 193-194, 2009, p. 73-74. En ligne [consulté le 28 février 2017] : https://etudesafricaines.revues.org/18638

Anne Doquet, «La force de l'impact. Paradigme théorique et réalités de terrain », Espacestemps.net , 2010. En ligne [consulté le 28 février 2017] : http://www.espacestemps.net/articles/forceimpact/

Anne Doquet, « Ethnologues et touristes au calque papier. Quelques effets de terrain en milieu touristique », Espacestemps.net, 2016. En ligne [consulté le 28 février 2017] : http:// www.espacestemps.net/articles/ethnologues-et-touristes-au-papier-calque/

Espacestemps.net, traverse 2010-2016 : « Le tourisme à l'épreuve de l'enquête » (dir. Saskia cousin). En ligne [consulté le 28 février 2017] : http://www.espacestemps.net/articles/le-tourisme-aepreuve-de-enquete/

Ethnologie française, vol. 32 (3) : « Touristes, autochtones : qui est l'étranger ?» (dir. André Rauch), 2002.

Ethnologies, vol. 32 (2) : « Tourisme culturel » (dir. Habid Saidi), 2010.

Nelson H. H. Graburn, Ethnic and Tourist Arts: Cultural Expressions from the Fourth World, University of California Press, 1976.

Nelson H. H. Graburn, « The past in the present in Japan: nostalgia and neo-traditionalism in contemporary Japanese domestic tourism », dans Richard Butler et Douglas Pearce (dir.), Changes in Tourism: People, Places Processes, Routledge, 1995, p. 47-70.

Nelson H. H. Graburn, « Secular ritual: a general theory of tourism », dans Valene Smith, Hosts and Guests Revisited: Tourism Issues of the $21^{\text {st }}$ Century, Cognizant Communication, 2001.

Marcel Griaule, « Mission ethnographique et linguistique Dakar-Djibouti », Journal de la Société des africanistes, vol. 1, nos 1-2, 1931, p. 113-122.

Jafar Jafari, « Entry into a new field of study: leaving a footprint », dans Denisson Nash (dir.), The Study of tourism: Anthropological and Sociological Beginnings, Elsevier, 2007, p. 108-121.

Marie-Françoise Lanfant, Les Théories du loisir. Sociologie du loisir et idéologies, Presses universitaires de France, 1972.

Marie-Françoise Lanfant (dir.), Sociologie du tourisme : positions et perspectives dans la recherche internationale, Centre d'études sociologiques, 1978

Caroline Legrand, « Tourisme des racines et confrontations identitaires dans l'Irlande des migrations ", Diasporas, histoire et sociétés, nº 8, 2006, p. 162-171.

Naomi Leite et Nelson H. H. Graburn, « Anthropological interventions in tourism studies », dans Jamal Tazim et Mike Robinson (dir.), The Sage Handbook of Tourism Studies, Sage, 2009, p. 35-64.

Naomi Leite et Nelson H. H. Graburn, «L'anthropologie pour étudier le tourisme », Mondes du tourisme, $n^{\circ} 1,2010$. En ligne [consulté le 28 février 2017] : https://tourisme.revues.org/316

Claude Lévi-Strauss, Tristes Tropiques, Plon, 1955.

Claude Lévi-Strauss, La Pensée sauvage, Plon, 1962.

Dean MacCannel, The Tourist: A New Theory of the Leisure Class, Schocken Books, 1972. 
Laetitia Merli, Shaman Tour, documentaire Arte, $63 \mathrm{~min}, 2009$. En ligne [consulté le 28 février 2017] : https://vimeo.com/129572056

Denisson Nash (dir.), The Study of tourism: Anthropological and Sociological Beginnings, Elsevier, 2007

Pàl Nyiri, Scenic Spots: Chinese Tourism, the State, and Cultural Authority, University of Washington Press, 2006.

Theron Nunez, « Tourism, tradition and acculturation : Weekendismo in a Mexican village », Ethnology, vol. 2, $\mathrm{n}^{\circ}$ 3, 1963, p. 347-352.

Kjell Olsen, « Authenticity as a concept in tourism research. The social organization of the experience of authenticity ", Tourist Studies, vol. 2, n 2, 2002, p. 159-182.

Johanne Pabion Mouriès, L'Écotourisme au Kirghizistan post-soviétique. Entre développement international et volontés locales, thèse de doctorat en anthropologie sociale, Écoles des hautes études en sciences sociales, 2010.

David Picard, Tourism, Magic and Modernity. Cultivating the Human Garden, Berghahn Books, 2011.

David Picard et Sonja Buchberger, Couchsurfing Cosmopolitanisms: Can Tourism Make a Better World? Transcript Verlag, 2013.

Michel Picard, Bali : Tourisme culturel et culture touristique, L'Harmattan, 1992.

Susan Carol Rogers, "Which heritage? Nature, culture, and identity in French rural tourism », French Historical Studies, vol. 25, n 3, 2002, p. 475-503.

Sébastien Roux, «De quelques dynamiques contemporaines en anthropologie du tourisme francophone ", Cahiers d'études africaines, vol. 193-194, 2009, p. 595-602. En ligne [consulté le 28 février 2017] : https://etudesafricaines.revues.org/18847

Sébastien Roux, No money, no honey. Économies intimes du tourisme sexuel en Thailande, La Découverte, 2011.

Noel Salazar, « Tourisfying Tanzania: Local guides, global discourse », Annals of Tourism Research, vol. 33, no 3, 2006, p. 833-852.

Noel Salazar, Envisioning Eden: Mobilizing Imaginaries in Tourism and Beyond, « New Directions in Antropology » (31), Berghahn, 2010.

Noel Salazar, « Imagineering otherness: anthropological legacies in contemporary tourism », Anthropological Quarterly, vol. 86, nº 3, p. 669-696, 2013.

Noel Salazar et Nelson H. H. Graburn (dir.), Tourism Imaginaries: Anthropological Approaches, Berghahn, 2014.

Christine Salomon, « Antiquaires et businessmen de la Petite Côte du Sénégal. Le commerce des illusions amoureuses ", Cahiers d'études africaines, vol. 193-194, 2009, p. 147-176. En ligne [consulté le 28 février 2017] : https://etudesafricaines.revues.org/18671

Tom Selwyn (dir.), The Tourist Image: Myths and Myth-Making in Tourism, John Wiley, 1996.

Valerio Simoni, «Shifting powers: the destabilization of asymmetries in the realm of tourism in Cuba », Tsansta. Review of the Swiss Anthropological Society, vol. 13, 2008, p. 11-19.

Valene Smith, Hosts and Guests: The Anthropology of Tourism, Blackwell, 1978.

Hasso Spode, « La recherche historique sur le tourisme. Vers une nouvelle approche », Mondes du tourisme, $\mathrm{n}^{\circ} 2,2010$, p. 4-18. En ligne [consulté le 28 février 2017] : https://

tourisme.revues.org/267 
Anne-Marie Thiesse, Écrire la France, Presses universitaires de France, 1991.

Anne-Marie Thiesse, La Création des identités nationales : Europe, XVIII ${ }^{e}-X X^{e}$ siècle, Seuil, 1999.

Jean-Didier Urbain, L'Idiot du voyage. Histoires de touristes, Payot, 1991.

Jean-Didier Urbain, Secrets de voyage : menteurs, imposteurs et autres voyageurs invisibles, Payot \& Rivages, 1998.

Jean-Didier Urbain, Ethnologue mais pas trop... Ethnologie de la proximité, voyages secrets et autres expéditions minuscules, Payot \& Rivages, 2003.

Thorstein Veblen, The Theory of the Leisure Class: An Economic Study of Institutions, Macmillan, 1899.

Jacquelin Waldren, Insiders and Outsiders: Paradise and Reality in Mallorca, « New Directions in Anthropology » (3), Berghahn, 1996.

Ning Wang, « Rethinking authenticity in tourism experience ", Annals of Tourism Research, vol. 26, $\mathrm{n}^{\circ} 2,1999$, p. 349-370.

Yves Winkin, Anthropologie de la communication. De la théorie au terrain, De Boeck - Seuil, 2001, «Le tourisme et son double », p. 206-224

\section{NOTES}

1. Merci à Mathis Stock et Inès Jorgensen pour leurs lectures, leurs remarques et leurs conseils.

2. On peut les compter sur les doigts d'une main : cinq emplois dont quatre femmes : deux postes à l'IRD (A. Doquet et $\mathrm{O}$. Evrad), trois à l'université (M. Boullosa, A. Condevaux et S. Cousin) sont explicitement liés au tourisme et à l'anthropologie, dont deux postes créés il y a moins de cinq ans).

3. C'est toutefois la revue québécoise Anthropologie et Sociétés qui inaugure ce cycle de numéros spéciaux de revue en 2001, avec le numéro "Tourisme et sociétés locales en Asie orientale ", dirigé par deux chercheurs spécialistes de l'Asie : Jean Michaud, chercheur à l'université de Hull (Royaume-Uni) et Michel Picard, chercheur au CNRS.

\section{ABSTRACTS}

This article aims to study the relationship between anthropology and tourism-as one of its objects. From the pionneers in the 1960s to the latest publications, the authors will examine the theoritical and ethnographical diversity of the approaches used in anthropology to study tourism. In a reflexive approach, it will trace the academical, scientifical and epistemological difficulties encountered by the anthropologist working in a field often despised in its own discipline. Furthermore, the article will explore how tourism itself could be approached as a social and cultural practice that is partially based on a reappropriation of anthropological knowledge. Ultimately, the authors suggest that tourism could be defined as an "alternity enonomy", which values depend on relations, practices or imaginations of alterity. 
Cet article propose une analyse des relations entre la discipline anthropologique et l'objet tourisme. Il relate la diversité des approches méthodologiques et théoriques depuis les pionniers des années 1960 jusqu'aux dernières publications. Dans une perspective réflexive, il retrace les difficultés académiques, scientifiques et épistémologiques que rencontre l'ethnologue face à une pratique sociale souvent méprisée par sa discipline. D'autre part, il se penche sur la manière dont le tourisme met et scène, en mots et en marché les savoirs ethnologiques. In fine, les auteurs proposent de définir et d'étudier le tourisme comme une économie de l'altérité, dont la valeur d'échange tient aux relations, aux pratiques ou aux imaginaires d'altérité générés.

\section{INDEX}

Mots-clés: Anthropologie, tourisme, altérité

Keywords: Anthropology, tourism, alterity

\section{AUTHORS}

\section{SASKIA COUSIN}

Anthropologue, maîtresse de conférences, université Paris Descartes / Canthel, membre de l'Institut universitaire de France,- saskia.cousin@parisdescartes.fr

\section{THOMAS APCHAIN}

Doctorant en anthropologie culturelle et sociale, université Paris Descartes / Canthel thomas.apchain@etu-parisdescartes.fr 